07

\title{
Установление стационарного состояния при наложении интенсивной пластической деформации кручения в двухкомпонентных кристаллах
}

\author{
() Ю.Д. Заворотнев ${ }^{1}$, Л.С. Метлов ${ }^{1,2}$, Е.Ю. Томашевская ${ }^{3}$ \\ ${ }^{1}$ Донецкий фозико-технический институт им. А.А. Галкина, \\ Донецк, Украина \\ 2 Донецкий национальный университет, \\ Донецк, Украина \\ ${ }^{3}$ Донецкий национальный университет экономики и торговли им. М. Туган-Барановского, \\ Донецк, Украина \\ E-mail: zavorotnev.yurii@mail.ru
}

Поступила в Редакцию 24 декабря 2021 г.

В окончательной редакции 24 декабря 2021 г.

Принята к публикации 27 декабря 2021 г.

\begin{abstract}
Рассмотрено распространение волны переключения (кинк) в двухкомпонентных кристаллах при наложении интенсивной пластической деформации кручения. При этом использовалась феноменологическая теория Ландау с учетом инвариантов Лифшица. Проведено численное моделирование формы кинка, возникающего при различных температурных воздействия на кристалл.
\end{abstract}

Ключевые слова: кинк, параметр порядка, кручение, потенциал, двухкомпонентный кристалл.

DOI: 10.21883/FTT.2022.04.52186.263

\section{1. Введение}

В настоящее время актуальным является вопрос поведения материалов при наложении интенсивной пластической деформации кручения (ИПДКР). Как показано в работах [1,2], при наложении ИПДКР может меняться фазовый состав двухкомпонентных материалов типа $\mathrm{Cu}-\mathrm{Ag}, \mathrm{Cu}-\mathrm{Sn}, \mathrm{Cu}-\mathrm{Co}$. При этом осуществлялся неразрушающий процесс воздействия кручения. Обнаружено, что при достаточно большом количестве оборотов система независимо от начального состояния и температуры отжига всегда приходит к одному и тому же стационарному состоянию. При этом фазовая граница диаграммы зависимости концентрации растворенной примеси от температуры отжига $T_{a n n}$ смещалась и вырождалась в прямую вертикальную линию [1]. Параллельно с этим синхронно происходило изменение параметров решетки. Подобное поведение наблюдаемых величин можно объяснить, опираясь на феноменологическую теорию Ландау $[3,4]$. Однако, в этих работах не рассматривалась форма фронта волны (кинк), возникающей при наложении ИПДКР. Изучению этого вопроса посвящена настоящая работа. Перемещение фронта является автоволновым процессом в форме кинка и является простейшим топологическим солитоном. После прохода фронта через весь кристалл устанавливается стационарное состояние. Автоволны возникают в самых различных средах физического, химического и биологического происхождения. Их примерами могут служить концентрационные волны в реакции Белоусова-Жаботинского [5], волны химической сигнализации в колониях некоторых микроорганизмов [6], волны в межзвездном газе, приводящие к образованию спиральных галактик [7]. Важный пример активных сред представляют многие биологические ткани. Так, автоволновую природу имеют распространение нервного импульса [8] и возбуждения в сердечной мышце [9]. В физических системах автомодельные решения дифференциального уравнения отвечают движению межфазной границы при фазовых переходах первого или второго рода $[10,11]$, описывают распространение пламени [12]. В вышеперечисленных работах находились аналитические приближенный решения. В [13] была предпринята попытка численного рассмотрения этой проблемы, позволяющая получить более точное решение. В настоящей работе идеи, развитые в $[3,4,13]$, применены к двухкомпонентным кристаллам.

\section{2. Теория}

Пусть на двухкомпонентный кристалл с одним закрепленным концом наложено ИПДКР в области фазовой диаграммы, в которой примесь полностью растворена. В настоящей работе рассмотрим поведение фронта (кинк) возникающей бегущей волны. В этом случае можно воспользоваться приближением виртуального кристалла. При его использовании предполагается, что все характеристики кристалла усредняются и можно ввести единый параметр порядка (ПП), описывающий деформацию элементарной ячейки. Для установления формы кинка воспользуемся феноменологической теорией Ландау.

Как показано в $[10,11]$, динамику кинка удобно рассматривать с помощью дифференциального уравнения 
типа Ландау-Халатникова [14]:

$$
\frac{\partial q_{i}}{\partial t}=-\gamma_{i i} \frac{\delta \widetilde{\Phi}}{\delta q_{i}} ; \quad(i=x, y),
$$

где $\widetilde{\Phi}-$ функционал свободной энергии,

$$
\frac{\delta \widetilde{\Phi}}{\delta q_{i}}=\sum_{k}(-1)^{k} \frac{d^{k}}{d z^{k}} \frac{\partial \Phi}{\partial\left(\frac{\partial^{k} q_{i}}{\partial z^{k}}\right)}
$$

- функциональная производная, $t-$ время, $\gamma_{i i}(i=x, y)-$ компоненты параметра, характеризующего скорость релаксации системы к положению равновесия. В дальнейшем будем предполагать, что эти компоненты постоянны и для простоты пренебрежем недиагональными компонентами. Уравнение ЛандауХалатникова является уравнением в частных производных относительно времени (левая часть) и пространственных переменных (правая часть) и описывает процесс релаксации из одного состояния в другое. Создание уравнения базировалось на том факте, что при $t \rightarrow \infty$ физическая система приходит в стационарное состояние. В зависимости от правой части этот переход может быть плавным, скачкообразным или через несколько промежуточных состояний. На вид перехода оказывают влияние дисперсия, диссипация, скорость релаксации и другие факторы. Уравнение Ландау-Халатникова допускает множество решений, зависящих от вида неравновесного термодинамического потенциала и величины его параметров. Это уравнение описывает процессы релаксации, как в идеальных кристаллах, так и в кристаллах с дефектами. Различные отклонения от идеальной структуры в неравновесном термодинамическом потенциале НТДП определяются с помощью производных различного порядка от соответствующих ПП по пространственным переменным. В частности, в кристаллах без центра инверсии длиннопериодические спиральные структуры можно описать с помощью инвариантов Лифшица. Если кристалл с центром инверсии, то необходимо в НТДП ввести квадраты первых и вторых производных. В последнем случая система уравнений (2) становится системой четвертого порядка относительно пространственных производных, что требует большого количества начальных (граничных) условий и решение усложняется. Поэтому в настоящей работе ограничимся кристаллами без центра инверсии.

Плотность НТДП-системы, которая подвергнута воздействию ИПДКР с направленным по оси $O Z$ моментом $M$, имеет вид $[3,4,13,15]$ :

$$
\begin{aligned}
\widetilde{\Phi} & =\frac{\alpha_{1}}{2} q^{2}(N)+\frac{\alpha_{2}}{4} q^{4}(N)+\frac{\alpha_{3}}{6} q^{6}(N)+\gamma_{1} M^{r} \\
& \times\left(q_{x} \frac{\partial q_{y}}{\partial z}-q_{y} \frac{\partial q_{x}}{\partial z}\right)+\gamma_{2} M^{s}\left(\left(\frac{\partial q_{x}}{\partial z}\right)^{2}+\left(\frac{\partial q_{y}}{\partial z}\right)^{2}\right) \\
& +\beta_{1} \varphi+\frac{\beta_{2}}{2} \varphi^{2}+\frac{\beta_{3}}{2} \varphi^{3}+\delta q^{2} \varphi
\end{aligned}
$$

где $\gamma_{i}(i=1,2), \alpha_{i}, \beta_{i}(i=1-3), \delta-$ феноменологические постоянные, $q$ - векторный структурный ПП с компонентами $q_{x}$ и $q_{y}$, который определяется как линейная комбинация сдвигов ионов элементарной ячейки в результате ФП, $\varphi$ - плотность дефектов. Компонента $q z$ не учитывается, поскольку момент кручения направлен по оси $O Z$. Фактически в данной работе рассматривается возможность возникновения только поперечных волн. Момент кручения $M$ является аксиальным вектором. Поэтому в кристаллах без центра инверсии степени $r$ и $s$ в (1) должны быть четными. Как показано в работе [13], $r=6, s=2$. В потенциале (1) не выписано слагаемое, ответственное за упругое взаимодействие. Процедура его исключения приведена в работе [16]. Необходимо отметить, что инварианты Лифшица, описывающие возникающую при наложении ИПДКР деформацию, в кристаллах с центром инверсии отсутствуют, поскольку возникающая аксиальная симметрия не снимает инверсию. Поэтому в данной работе рассматриваются кристаллы без центра инверсии. Подставляя (2) в (1) получаем

$$
\left\{\begin{array}{rl}
\frac{\partial q_{x}}{\partial t}= & -\gamma_{x x}\left[q_{x}\left(\alpha_{1}+\alpha_{2} q^{2}+\alpha_{3} q^{4}+2 \delta \varphi\right)\right. \\
& \left.+2 \gamma_{1} M^{r} \frac{\partial q_{y}}{\partial z}-2 \gamma_{2} M^{s} \frac{\partial^{2} q_{x}}{\partial z^{2}}\right] \\
\frac{\partial q_{y}}{\partial t}= & -\gamma_{y y}\left[q_{y}\left(\alpha_{1}+\alpha_{2} q^{2}+\alpha_{3} q^{4}+2 \delta \varphi\right)\right. \\
& \left.-2 \gamma_{1} M^{r} \frac{\partial q_{x}}{\partial z}-2 \gamma_{2} M^{s} \frac{\partial^{2} q_{y}}{\partial z^{2}}\right]
\end{array} .\right.
$$

Как показано в $[3,4]$, величина плотности дефектов $\varphi$ зависит от температуры отжига $T_{a n n}$. Поскольку соответствующий закон не известен, то искомая зависимость подбиралась методом наилучшего согласия теоретических и экспериментальных данных. Поскольку зависимость слабая, то в настоящей работе ею пренебрегаем. Полученная система уравнений является автономной и ее можно существенно упростить путем перехода к автомодельной переменной

$$
u=z-c t,
$$

где $c-$ фазовая скорость распространения волны. Математически данное преобразование означает переход к движущейся системе координат, в которой волна неподвижна, и при этом распределение характеристик в разные моменты времени получаются путем сдвига. Ясно, что в этом случае класс решений системы сужается и существенно упрощается нахождение решения. В итоге получаем систему обыкновенных дифференциальных 
уравнений

$$
\left\{\begin{array}{c}
2 \gamma_{x x} \gamma_{2} M^{s} \frac{d^{2} q_{x}}{d u^{2}}-2 \gamma_{x x} \gamma_{1} M^{r} \frac{d q_{y}}{d u}+c \frac{d q_{x}}{d u} \\
=\gamma_{x x} q_{x}\left(\alpha_{1}+\alpha_{2} q^{2}+\alpha_{3} q^{4}+2 \delta \varphi\right) \\
2 \gamma_{y y} \gamma_{2} M^{s} \frac{d^{2} q_{y}}{d u^{2}}+2 \gamma_{y y} \gamma_{1} M^{r} \frac{d q_{x}}{d u}+c \frac{d q_{y}}{d u} \\
=\gamma_{y y} q_{y}\left(\alpha_{1}+\alpha_{2} q^{2}+\alpha_{3} q^{4}+2 \delta \varphi\right)
\end{array}\right.
$$

Для решения этой системы необходимо задать четыре условия, в качестве которых можно взять $q_{x}(-\infty)$, $q_{y}(-\infty), q_{x}(+\infty), q_{y}(+\infty)$. Поскольку при $t=0$ и произвольном значении $z(u=z)$ система находится в равновесном состоянии (ИПДКР отсутствует), то все производные равны нулю. Следовательно, величина $q(+\infty)$ определяются из системы алгебраических уравнений

$$
\alpha_{1}+\alpha_{2} q^{2}+\alpha_{3} q^{4}+2 \delta \varphi=0
$$

Ввиду неопределенности в настоящей работе принято $q_{x}(+\infty)=q_{y}(+\infty)=\frac{q(+\infty)}{\sqrt{2}}$.

В работах $[3,4]$ показано, что величины коэффициентов $\alpha_{i}(i=1-3)$ зависят от температуры отжига и, поэтому стационарные состояния будут несколько отличаться друг от друга. Однако для простоты в настоящей работе этим незначительным различием пренебрегаем. При $t=+\infty(u=-\infty)$ система также находится в равновесном состоянии и под воздействием ИПДКР в ней имеется кристаллическая спиральная структура, которая описывается соотношениями $q_{x}=q(-\infty) \cdot \cos (k \cdot z), q_{y}=q(-\infty) \cdot \sin (k \cdot z)$. Здесь модуль ПП является постоянной величиной. Для нахождения следующей пары граничных условий необходимо подставить эти соотношения в (3) с учетом того, что все производные по времени равны нулю. После несложных преобразований получается биквадратное алгебраическое уравнение, решение которого имеет вид [3]:

$$
q^{2}(-\infty)=\frac{-\alpha_{2}+\sqrt{\alpha_{2}^{2}+4 \alpha_{3}\left(\alpha_{1}-2 \delta \varphi+\frac{\gamma_{1}^{2} M^{2 r-s}}{2 \gamma_{2}}\right)}}{2 \alpha_{3}}
$$

Здесь также принимаем $q_{x}(-\infty)=q_{y}(-\infty)=\frac{q(-\infty)}{\sqrt{2}}$. Как показано в [1-4], в двухкомпонентных системах типа $\mathrm{Cu}-A(A=\mathrm{Ag}, \mathrm{Sn}, \mathrm{Co})$ имеет место критическая температура отжига $T_{k r a n n}$, при переходе через которую поведение системы кардинально меняется. При $T_{a n n}<T_{k r \text { ann }}$ функция $q(N)$, где $N$ - число оборотов возрастающая и выпуклая, при $T_{a n n}>T_{k r a n n}$ искомая зависимость - убывающая и вогнутая. Подобное поведение объясняется расходимостью и изменением знака коэффициента $\gamma_{2}$ в окрестности точки $T_{a n n}<T_{k r \text { ann }}[3,4]$. Эта особенность влияет и на форму кинка.

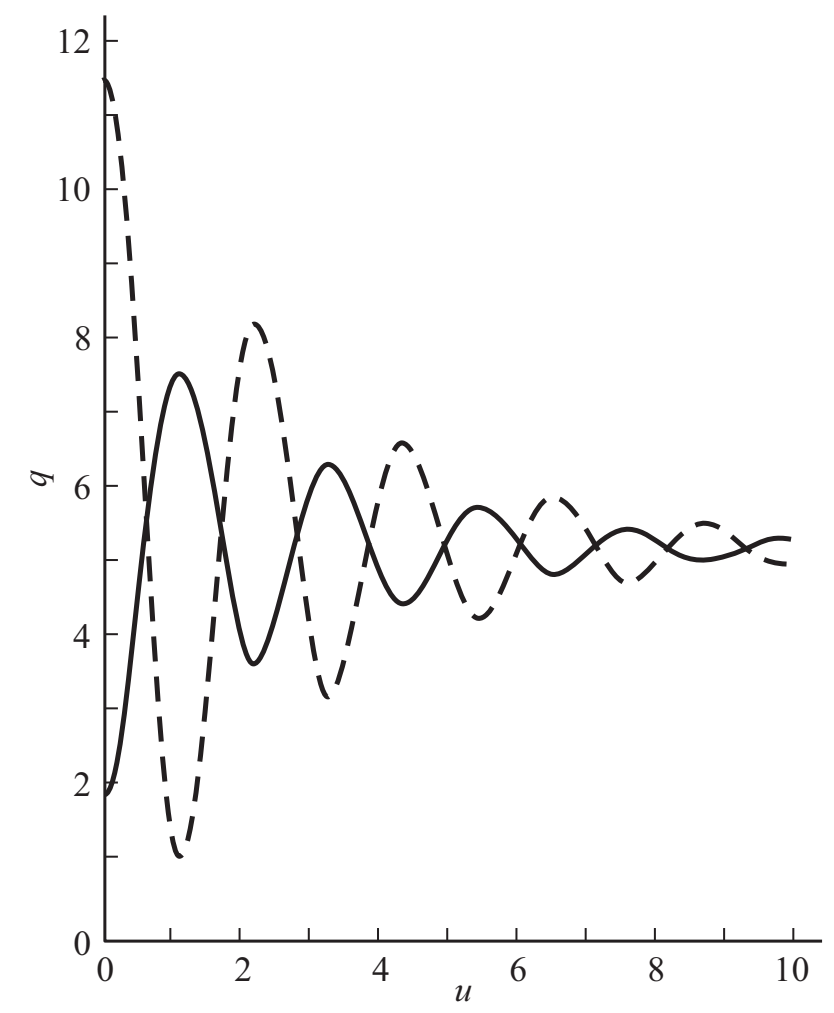

Рис. 1. Качественная форма кинка при малой скорости. По осям приняты произвольные единицы измерения.

На рис. 1 изображен устоявшийся режим распространения для достаточно больших времен после воздействия ИПДКР и представлена зависимость $q(u)$ для случая $T_{a n n}<T_{k r a n n}$ (сплошная кривая) и $T_{a n n}>T_{k r a n n}$ (пунктирная кривая). В обоих случаях имеет место колебательный процесс при переходе из одного состояния в другое. Эта особенность переднего фронта кинка зависит от его скорости и величины коэффициента релаксации. Увеличение скорости распространения с приводит к увеличению затухания колебательного процесса, т.е. уменьшается амплитуда и количество колебание до перехода в равновесное состояние. В пределе большой скорости форма кинка становится монотонной (убывающая или возрастающая). При увеличении коэффициента релаксации растут амплитуды колебаний переднего фронта и их количество. Уменьшение $\gamma$ действует аналогично увеличению невозмущенной скорости.

Уменьшение значения коэффициента $\gamma_{2}$ приводит к увеличению частоты затухающих колебаний и не влияет на их амплитуду. При этом происходит более быстрый переход в конечное стационарное состояние (рис. 2). Необходимо отметить, что аналогичные осциллирующие хвосты солитонов были предсказаны в работе [17].

На поведение переднего фронта может оказывать влияние дисперсия, величина которой определяется постоянной $\gamma_{1}$. Предположим, что причиной появления слабой дисперсии является изменение величины ПП. 


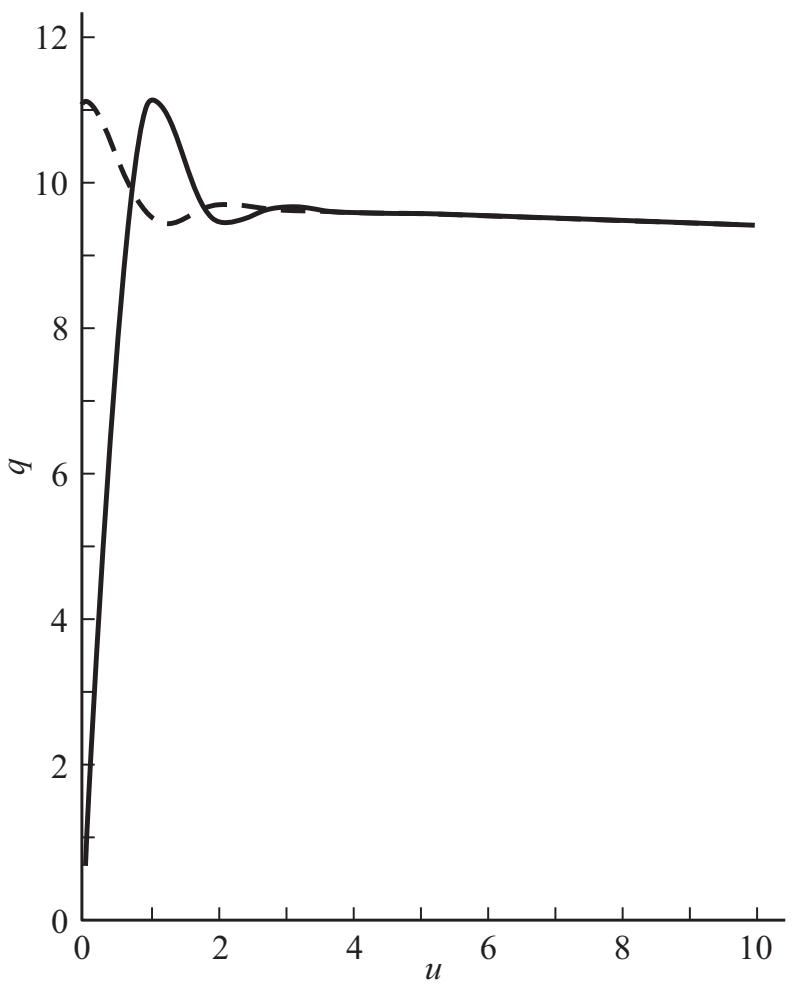

Рис. 2. Качественная форма кинка при большой скорости. Сплошная линия $-T_{a n n}<T_{k r a n n}$. Пунктирная линия $T_{a n n}>T_{k r a n n}$. По осям приняты произвольные единицы измерения.

Тогда $\gamma_{1}$ можно разложить в ряд по степеням ПП. Рассмотрим два случая.

1. Квадратичная зависимость: $\quad \gamma_{1}=\gamma_{10}+\gamma_{11}$ $\times(q-q(+\infty))^{2}$. Соответствующая система автоволновых уравнений имеет вид

$$
\left\{\begin{array}{l}
2 \gamma_{x x} \gamma_{2} M^{s} \frac{d^{2} q_{2 x}}{d u^{2}}-2 \gamma_{x x}\left(\gamma_{10}+\gamma_{11}\left(q_{2}-q_{2}(+\infty)\right)^{2}\right. \\
\left.\quad+2 \gamma_{11}\left(q_{2 x}-q_{2 x}(+\infty)\right)\right) M^{r} \frac{d q_{2 y}}{d u}+\left(c+2 \gamma_{x x} \gamma_{11} M^{s}\right. \\
\left.\quad \times\left(q_{2 x}-q_{2 x}(+\infty)\right)\left(q_{2 y}-q_{2 y}(+\infty)\right)\right) \frac{d q_{2 x}}{d u} \\
=\gamma_{x x} q_{2 x}\left(\alpha_{1}+\alpha_{2} q_{2}^{2}+\alpha_{3} q_{2}^{4}+2 \delta \varphi\right) \\
2 \gamma_{y y} \gamma_{2} M^{s} \frac{d^{2} q_{2 y}}{d u^{2}}+2 \gamma_{y y}\left(\gamma_{10}+\gamma_{11}\left(q_{2}-q_{2}(+\infty)\right)^{2}\right. \\
\left.\quad+2 \gamma_{11}\left(q_{2 y}-q_{2 y}(+\infty)\right)\right) M^{r} \frac{d q_{2 x}}{d u}+\left(c+2 \gamma_{y y} \gamma_{11} M^{s}\right. \\
\left.\quad \times\left(q_{2 x}-q_{2 x}(+\infty)\right)\left(q_{2 y}-q_{2 y}(+\infty)\right)\right) \frac{d q_{2 y}}{d u} \\
=\gamma_{y y} q_{2 y}\left(\alpha_{1}+\alpha_{2} q_{2}^{2}+\alpha_{3} q_{2}^{4}+2 \delta \varphi\right)
\end{array}\right.
$$

График численного решения этой системы при учете начальных условий (6) и (7) приведен на рис. 3 пунк- тирной линией. Здесь же сплошной линией приведена форма кинка $\left(q_{1}(u)=q(u)\right)$ при $\gamma_{1}=$ const. Видно, что имеет место значительное уменьшение максимумов переднего фронта. Этот вывод подтверждается приведенными на рис. 4 графиками соответствующих скоростей

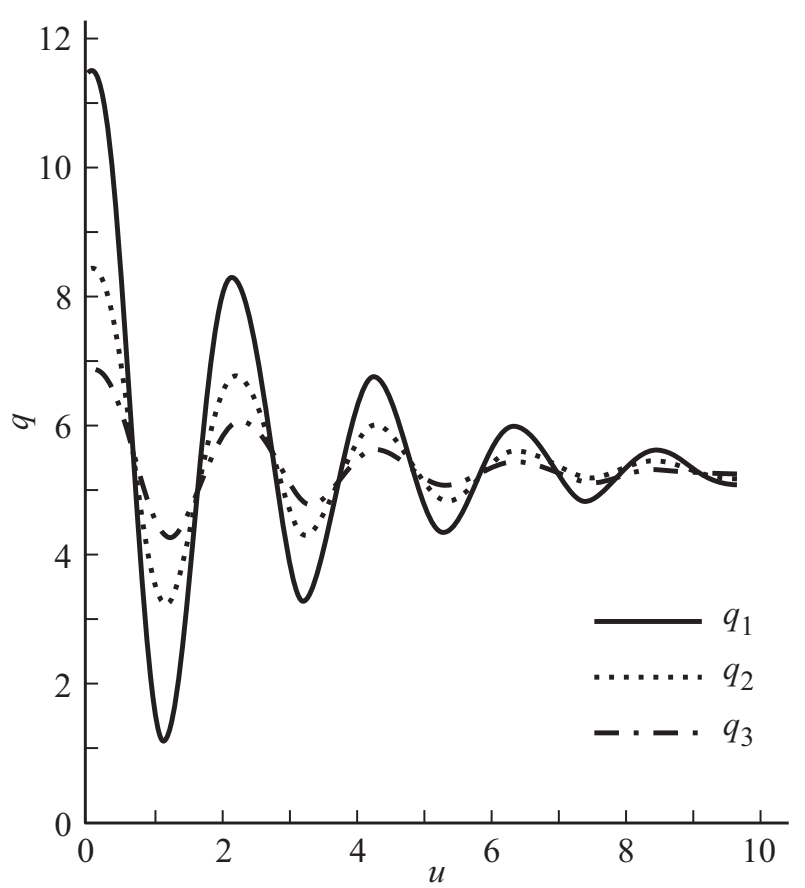

Рис. 3. Сравнение качественных форм кинка при квадратичной (пунктирная линия) и линейной (штрихпунктирная линия) зависимостей дисперсии от ПП. Сплошная линия - дисперсия отсутствует. По осям приняты произвольные единицы измерения.

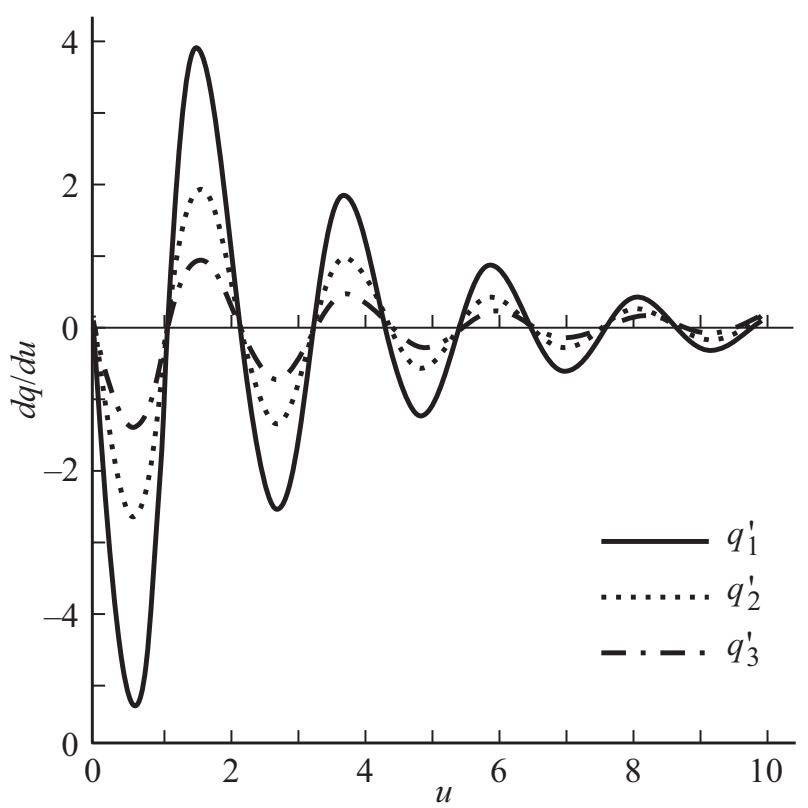

Рис. 4. Качественная форма производных ПП при квадратичной (пунктирная линия) и линейной (штрихпунктирная линия) зависимостей дисперсии. Сплошная линия - дисперсия отсутствует. По осям приняты произвольные единицы измерения. 
смещения ПП. Необходимо отметить, что качественное поведение ПП в правой части переднего фронта кинка в обоих случаях практически одинаково.

2. Линейная зависимость: $\gamma_{1}=\gamma_{10}+\gamma_{11}(q-q(+\infty))$. Система уравнений

$$
\left\{\begin{array}{l}
2 \gamma_{x x} \gamma_{2} M^{s} \frac{d^{2} q_{3 x}}{d u^{2}}-2 \gamma_{x x}\left(\gamma_{10}+\gamma_{11}\left(q_{3}-q_{3}(+\infty)\right)\right. \\
\left.\quad+\gamma_{11} \frac{\left(q_{3 x}-q_{3 x}(+\infty)\right)^{2}}{2\left(q_{3}-q_{3}(+\infty)\right)}\right) M^{r} \frac{d q_{3 y}}{d u}+\left(c+\gamma_{x x} \gamma_{11} M^{s}\right. \\
\left.\quad \times \frac{\left(q_{3 x}-q_{3 x}(+\infty)\right)\left(q_{3 y}-q_{3 y}(+\infty)\right)}{q_{3}-q_{3}(+\infty)}\right) \frac{d q_{3 x}}{d u} \\
=\gamma_{x x} q_{3 x}\left(\alpha_{1}+\alpha_{2} q_{3}^{2}+\alpha_{3} q_{3}^{4}+2 \delta \varphi\right) \\
2 \gamma_{y y} \gamma_{2} M^{s} \frac{d^{2} q_{3 y}}{d u^{2}}+2 \gamma_{y y}\left(\gamma_{10}+\gamma_{11}\left(q_{3}-q_{3}(+\infty)\right)\right. \\
\left.\quad+\gamma_{11} \frac{\left(q_{3 y}-q_{3 y}(+\infty)\right)}{2\left(q_{3}-q_{3}(+\infty)\right)}\right) M^{r} \frac{d q_{3 x}}{d u}+\left(c+\gamma_{y y} \gamma_{11} M^{s}\right. \\
\left.\quad \times \frac{\left(q_{3 x}-q_{3 x}(+\infty)\right)\left(q_{3 y}-q_{3 y}(+\infty)\right)}{q_{3}-q_{3}(+\infty)}\right) \frac{d q_{3 y}}{d u} \\
= \\
\gamma_{y y} q_{3 y}\left(\alpha_{1}+\alpha_{2} q_{3}^{2}+\alpha_{3} q_{3}^{4}+2 \delta \varphi\right)
\end{array}\right.
$$

На рис. 3, 4 штрихпунктирными линиями приведены найденные численным методом зависимости фронта кинка $\left(q_{3}(u)\right)$ и соответствующей производной. В правой половине переднего фронта имеет место уменьшение амплитуд колебания ПП, что приводит к меньшему значению ПП конечного стационарного состояния. Производные в правой части переднего фронта также имеют одинаковую форму.

Необходимо отметить, что в точке $u=0$ производные во всех случаях равны нулю, т.е. имеет место минимум колебательного процесса по отношению к конечному состоянию. Эта точка является устойчивой, поскольку первая производная от ПП равна нулю и обеспечивается минимум энергии. Аналогичная ситуация имеет место в задаче распространения теплового фронта [17]. В этой работе показано, что локальный максимум в начальной точке возможен только в системах с плоской симметрией, что имеет место в нашей задаче. Если симметрия осевая или шаровая, то в точке $u=0$ первая производная отлична от нуля.

\section{3. Выводы}

1. Показано, что при малой скорости распространения кинка в переднем фронте возникают колебания с возрастающей амплитудой.

2. Если скорость распространения кинка велика, то передний фронт представляет собой монотонную кривую.
3. Дисперсия оказывает существенное влияние на форму кинка. При линейной и квадратической дисперсии уменьшаются амплитуды колебаний, но частотный характер не меняется.

\section{Конфликт интересов}

Авторы заявляют, что у них нет конфликта интересов.

\section{Список литературы}

[1] B.B. Straumal, A.R. Kilmametov, Y. Ivanisenko, L. Kurmanaeva, B. Baretzky, Y.O. Kucheev, P. Zieba, A. Korneva, D.A. Molodov. Mater. Lett. 118, 111 (2014).

[2] B.B. Straumal, A.R. Kilmametov, B. Baretzky, O.A. Kogtenkova, P.B. Straumal, L. Litynska-Dobrzynska, R. Chulist, A. Korneva, P. Zieba. Acta Mater. 195, 184 (2020).

[3] B. Straumal, A. Kilmametov, A. Korneva, P. Zieba, Yu. Zavorotnev, L. Metlov, O. Popova, B. Baretzky. Crystals 11, 766 (2021).

[4] A. Korneva, A. Kimametov, Yu. Zavorotnev, L. Metlov, O. Popova, B. Baretzky. Mater. Lett. 302, 130386 (2021).

[5] A.N. Zaikin, A.M. Zhabotinsky. Nature 225, 535 (1970).

[6] F. Alcantara, M. Monk. Microbiology. 85, 321 (1974).

[7] B.F. Madore, W.L. Freedman. Am. Sci. 75, 252 (1987).

[8] N.A. Gorelova, J. Bures. J. Neurobiol. 14, 353 (1987).

[9] R.A. Gray, J. Jalife. Int. J. Bifurcation Chaos 06, 415 (1996).

[10] В.Н. Кащеев. Автомодельные решения эволюционного уравнения диффузионного типа. Препринт ЛАФИ-098. Саласпилс (1986). 21с.

[11] В.Н. Кащеев. Кинки при структурных фазовых переходах в системах с многокомпонентным параметром порядка. Препринт ЛАФИ-107. Саласпилс (1987). 22 с.

[12] Я.Б. Зельдович, Г.И. Баренблат, В.Б. Либрович, Г.М. Махвиладзе. Математическая теория горения и взрыва. Наука. M. (1980). $478 \mathrm{c}$.

[13] Yu.D. Zavorotnev, L.S. Metlov, A.Yu. Zakharov, E.Yu. Tomashevska. IOP Conf. Ser.: J. Phys.: Conf. Ser. 1352012063 (2019).

[14] Е.М. Лифшиц, Л.П. Питаевский. Физическая кинетика. Наука, М. (1979). 528 с.

[15] Ю.Д. Заворотнев., А.Ю. Захаров, Л.С. Метлов. ФТТ 59, 2266 (2017).

[16] Yu.D. Zavorotnev, E.H. Pashinskaya, V.N. Varjuchin, O.Yu. Popova. J. Magn. Magn. Mater. 349, 244 (2014).

[17] V.I. Karpman, E.M. Maslov. Sov. Phys. JETP 48, 252 (1978).

[18] П.П. Волосевич, Е.И. Леванов. Автомодельные решения задач газодинамики и теплопереноса. Изд-во МФТИ, М. (1997). 233 c.

Редактор Т.Н. Василевская 